\title{
Macroprolactinoma causing VI, X, XII cranial nerve palsies nearly 30 years after initial treatment
}

\author{
Anne de Bray1,2,3, Zaki K Hassan-Smith1,2, Jamal Dirie1,2, Edward Littleton4, Swarupsinh Chavda5, John Ayuk,2, \\ Paul Sanghera ${ }^{6}$ and Niki Karavitaki1,2,3 \\ 1Department of Endocrinology, Queen Elizabeth Hospital Birmingham, University Hospitals Birmingham NHS \\ Foundation Trust, Birmingham, UK, ${ }^{2}$ Centre for Endocrinology, Diabetes \& Metabolism, Birmingham Health Partners, \\ Birmingham, UK, ${ }^{3}$ Institute for Metabolism and Systems Research, University of Birmingham, Birmingham, UK, \\ ${ }^{4}$ Departments of Neurology, ${ }^{5}$ Radiology, and ${ }^{6}$ Oncology, Queen Elizabeth Hospital Birmingham, University Hospitals \\ Birmingham NHS Foundation Trust, Birmingham, UK
}

Correspondence should be addressed to $\mathrm{N}$ Karavitaki

Email

n.karavitaki@bham.ac.uk

\section{Summary}

A 48-year-old man was diagnosed with a large macroprolactinoma in 1982 treated with surgery, adjuvant radiotherapy and bromocriptine. Normal prolactin was achieved in 2005 but in 2009 it started rising. Pituitary MRIs in 2009, 2012, 2014 and 2015 were reported as showing empty pituitary fossa. Prolactin continued to increase (despite increasing bromocriptine dose). Trialling cabergoline had no effect (prolactin 191,380 mU/L). In January 2016, he presented with right facial weakness and CT head was reported as showing no acute intracranial abnormality. In late 2016, he was referred to ENT with hoarse voice; left hypoglossal and recurrent laryngeal nerve palsies were found. At this point, prolactin was $534,176 \mathrm{mU} / \mathrm{L}$. Just before further endocrine review, he had a fall and CT head showed a basal skull mass invading the left petrous temporal bone. Pituitary MRI revealed a large enhancing mass within the sella infiltrating the clivus, extending into the left petrous apex and occipital condyle with involvement of the left Meckel's cave, internal acoustic meatus, jugular foramen and hypoglossal canal. At that time, left abducens nerve palsy was also present. CT thorax/abdomen/ pelvis excluded malignancy. Review of previous images suggested that this lesion had started becoming evident below the fossa in pituitary MRI of 2015. Temozolomide was initiated. After eight cycles, there is significant tumour reduction with prolactin $1565 \mathrm{mU} / \mathrm{L}$ and cranial nerve deficits have remained stable. Prolactinomas can manifest aggressive behaviour even decades after initial treatment highlighting the unpredictable clinical course they can demonstrate and the need for careful imaging review.

\section{Learning points:}

- Aggressive behaviour of prolactinomas can manifest even decades after first treatment highlighting the unpredictable clinical course these tumours can demonstrate.

- Escape from control of hyperprolactinaemia in the absence of sellar adenomatous tissue requires careful and systematic search for the anatomical localisation of the lesion responsible for the prolactin excess.

- Temozolomide is a valuable agent in the therapeutic armamentarium for aggressive/invasive prolactinomas, particularly if they are not amenable to other treatment modalities. 


\section{Background}

Dopamine agonists (DAs) are an effective treatment for patients with macroprolactinoma leading to normalisation of prolactin (PRL) and tumour shrinkage in $66-100 \%$ and $64-96 \%$ of the cases, respectively (1). Secondary resistance to these agents is rare and is often manifested with substantial increases in PRL levels and tumour enlargement (2).

In cases non-responsive to DAs, the most commonly applied management options include changing to another DA, surgery and radiotherapy (alone or in combination) (3). In the last years, temozolomide has been added in the therapeutic armamentarium for unresponsive to conventional treatments prolactinomas (4).

We herein report a rare case of an 81-year-old male with a history of macroprolactinoma previously treated by surgery, radiotherapy and DA, which more than 20 years after initial therapy and after having achieved normal PRL, demonstrated aggressive behaviour with significant hyperprolactinaemia and uncommon symptomatology (left recurrent laryngeal, hypoglossal and abducens nerve palsies) due to tumour invasion. In this patient, treatment with temozolomide led to biochemical and imaging response.

\section{Case presentation}

In 1982, a 48-year-old man was admitted to hospital with worsening headaches (over 4 months) and diplopia (noticed 2 days prior to admission). Ophthalmology review showed reduced visual acuity bilaterally, left III cranial nerve palsy and papilloedema. Plain skull $X$ ray reported 'destruction of the pituitary fossa and erosion of the left sphenoid wing'. CT of head disclosed a 'middle fossa tumour occupying the tentorial hiatus and medial edge of the tentorium'. He was then transferred to the neurosurgical ward where it was decided to undergo craniotomy for urgent decompression and for establishing a pathological diagnosis. Based on the pathology report, histological diagnosis was not certain and the specimen could represent a pituitary tumour. At that time, the neurosurgical team referred the patient for radiotherapy, which was completed 2 months later. Involvement of the endocrine team took place after these interventions and serum prolactin was found at $600,000 \mathrm{mU} / \mathrm{L}$; furthermore, review of the pathology slides confirmed a chromophobe adenoma with positive immunostaining for PRL. The patient was started on bromocriptine on a final dose of $7.5 \mathrm{mg}$ daily, which led to a dramatic reduction of the PRL within a few months and levels of $2,500 \mathrm{mU} / \mathrm{L}$
1.5 years later. He remained under long-term follow-up and was maintained on bromocriptine $(7.5 \mathrm{mg}$ daily) and replacement with testosterone, hydrocortisone and levothyroxine. Normal serum PRL was finally achieved in 2005 (109 mU/L, reference range: $40-360 \mathrm{mU} / \mathrm{L})$ whilst on bromocriptine $2.5 \mathrm{mg}$ daily (on this dose for around 1 year), but this began to rise in $2009(2,421 \mathrm{mIU} / \mathrm{L})$. The patient had confirmed compliance with the DA treatment.

At around this time, his prostate-specific antigen (PSA) also began to rise. He was referred to the urology team who diagnosed prostatic adenocarcinoma (Gleeson score $3+3)$. He was placed under active surveillance and testosterone therapy was stopped.

\section{Investigations}

Pituitary MRIs performed in 2009, 2012, 2014 and 2015 were reported as showing empty pituitary fossa. However, serum PRL continued to rise significantly (despite increasing the dose of bromocriptine) reaching the value of $108,274 \mathrm{mU} / \mathrm{L}$ in 2015 . Trialling cabergoline (up to $3 \mathrm{mg}$ weekly) had no effect with the PRL measured at $191,380 \mathrm{mU} / \mathrm{L}$ a few months later.

In January 2016, he presented to the Emergency Department with right facial weakness and CT head was reported as showing 'no acute intracranial abnormality'. In a subsequent neurology review, the diagnosis of a transient ischaemic attack was thought unlikely but concerns were raised about the presence of manifestations of cerebellar ataxia. Review of the brain images, suggested significant cerebellar atrophy and the most likely cause of the ataxia was then considered to be the previous radiotherapy.

In late 2016, he was referred to an ENT team with a hoarse voice and was found to have left hypoglossal and left recurrent laryngeal nerve palsy. The possibilities of prolactinoma extension to the skull base or a new skull base tumour were raised. At this point, his serum PRL had reached a peak of $534,176 \mathrm{mU} / \mathrm{L}$ (on bromocriptine $15 \mathrm{mg}$ daily) but just before further endocrine review and imaging were performed, he had a fall for which he had CT head. This disclosed a basal skull mass invading the left petrous temporal bone. A subsequent pituitary MRI showed a large enhancing mass lesion within the sella infiltrating the clivus, extending into the left petrous apex and occipital condyle with involvement of the left Meckel's cave, internal acoustic meatus, jugular foramen and hypoglossal canal (Figs 1 and 2). At that time, left abducens nerve palsy had been added in his symptomatology. A CT thorax, abdomen and pelvis excluded malignancy and 

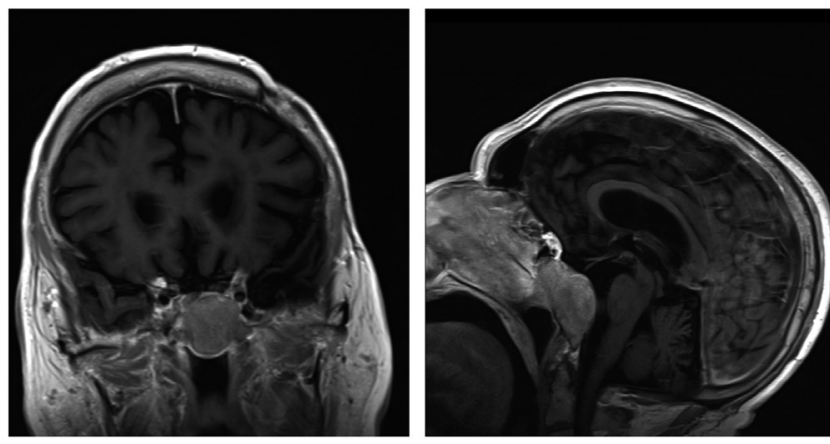

Figure 1

June 2017, Coronal and sagittal post-contrast T1 images showing mass infiltrating the clivus with extension into the left petrous apex and occipital condyle.

throughout this period, his PSA was undetectable. Review of previous images suggested that this lesion had started becoming evident below the fossa in the pituitary MRI performed in 2015 (as an area of low attenuation in the clivus, which in the latest MRI had increased around three times in maximum diameter). An invasive prolactinoma demonstrating aggressive behaviour and not responding to DA treatment was considered the diagnosis in this case.

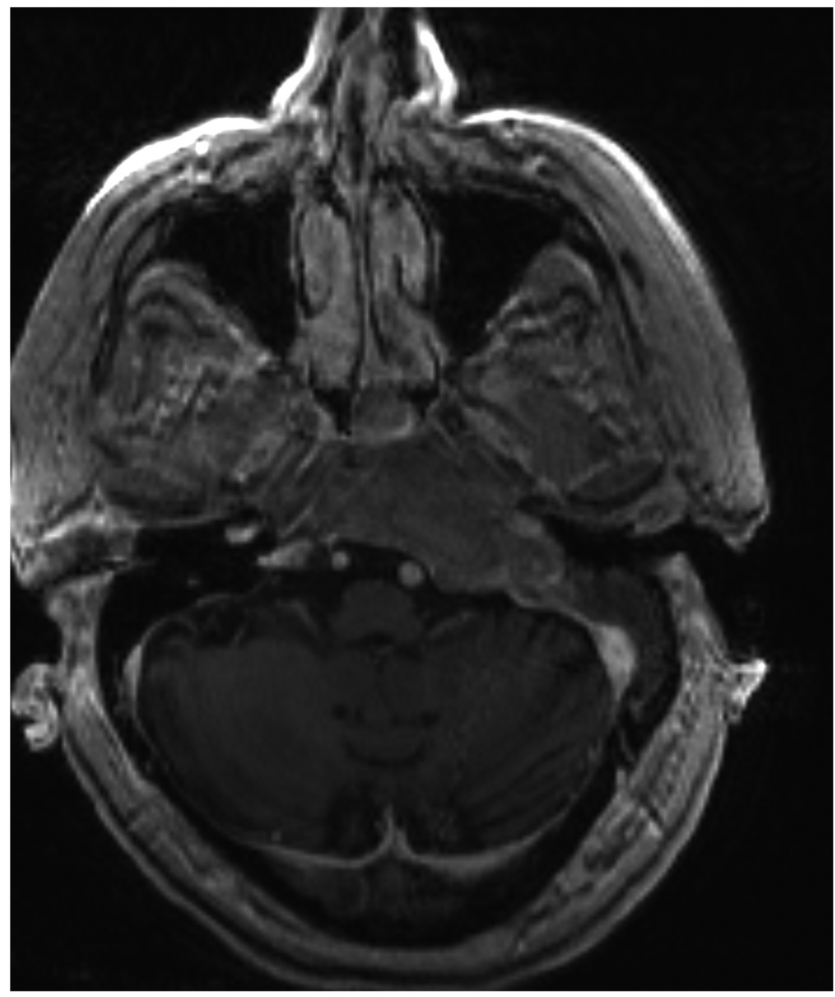

Figure 2

June 2017, Axial post-contrast T1 images. Note lateral extension into the clivus and occiput.

\section{Treatment}

Following discussion of the patient's case in the Pituitary Multidisciplinary Team meeting and taking into account the imaging appearances of the tumour, the presence of ataxia and the performance status of the patient (PS 2 ), surgery and repeat radiotherapy were not considered appropriate management options and treatment with temozolomide was suggested. This was initiated in July 2017 (150 mg/m² per os for 5 days increasing to $200 \mathrm{mg} / \mathrm{m}^{2}$ repeated every 4 weeks).

\section{Outcome and follow-up}

After three cycles of temozolomide, pituitary MRI demonstrated tumour volume reduction (Figs 3 and 4), and his serum PRL had fallen to 23,132 mIU/L. His cranial nerve deficits have remained stable and he is tolerating temozolomide well, with his main side effect being fatigue.

He is under regular oncology, endocrinology and neurology follow-up and his latest PRL (after nine cycles) was at $1565 \mathrm{U} / \mathrm{L}$. A total of 12 temozolomide cycles have been planned. Further decisions on extending the duration of treatment will be taken after the completion of the planned therapy and the review of tumour response.

\section{Discussion}

We have presented a rare case of a large macroprolactinoma treated with surgery, radiotherapy and DA which 27 years after initial therapy and 4 years after the achievement of normal PRL showed escape from control with a
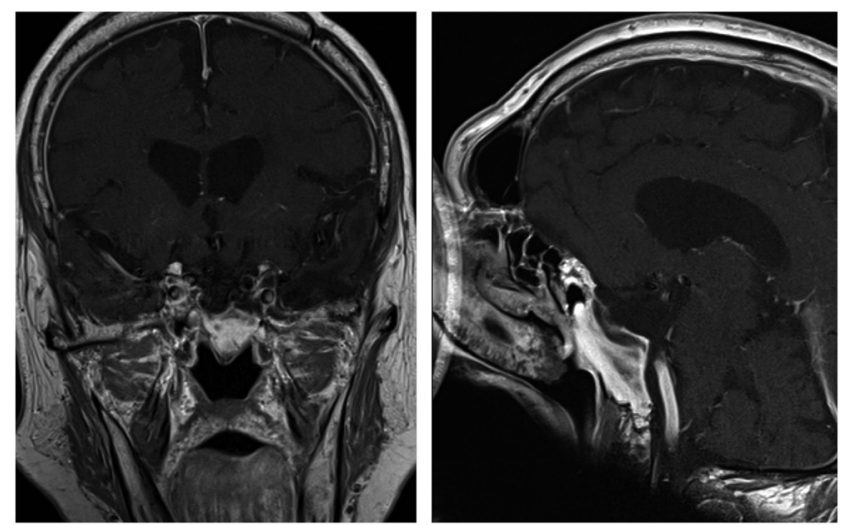

Figure 3

October 2017, Coronal and sagittal post-contrast T1 images after three cycles of temozolomide showing significant response to treatment on residual disease involving clivus, left petrous apex Meckel's cave, jugular foramen and hypoglossal canal. 


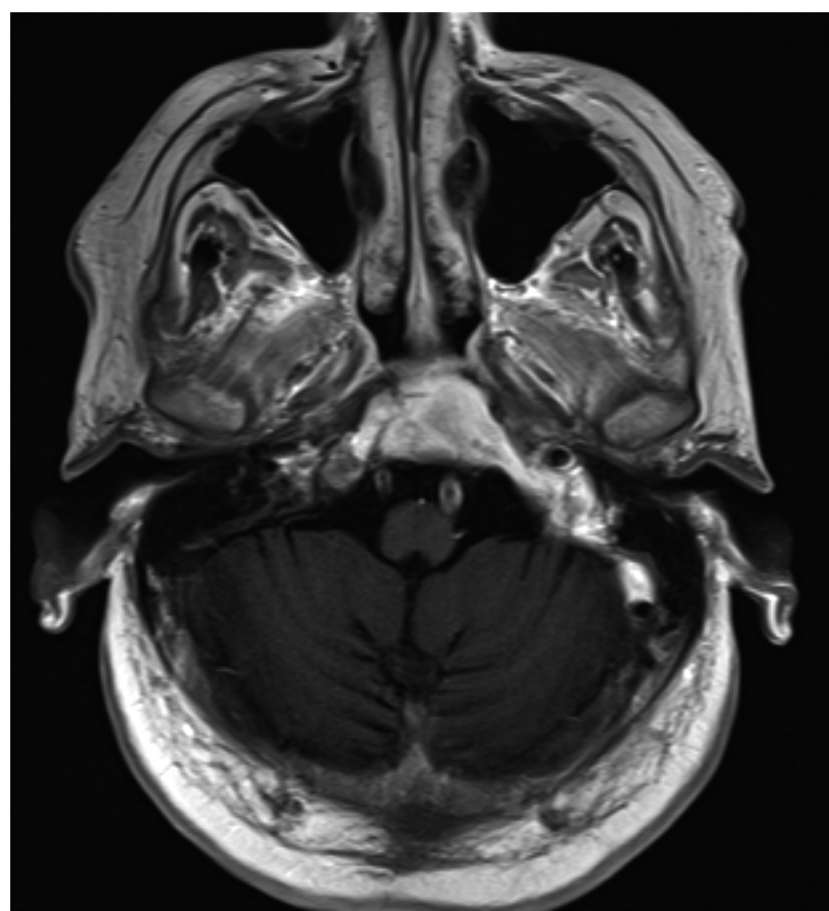

Figure 4

October 2017, Axial post-contrast T1 images after three cycles of temozolomide.

continuous dramatic rise in the PRL levels and invasion of surrounding structures causing palsies of left VI, X, XII cranial nerves. The tumour was not responsive to DA treatment and temozolomide was initiated with good biochemical/imaging response.

In our case, prolactinoma control was initially achieved following a multimodality approach. In a multicentre study of 92 patients with DA-resistant prolactinomas offered various treatment approaches (DA, surgery, radiotherapy), normal PRL was observed in $28 \%$ (26 out of 92 patients; 8 treated exclusively with DA and 18 with DA and surgery or DA and surgery and radiotherapy), adenoma disappearance in $18 \%$, and adenoma progression in $15 \%$ of the cases during a median follow-up period of 88 months (3). Overall, $4 \%$ of the patients had locally aggressive tumours and 3\% developed pituitary carcinoma (with the metastatic disease diagnosed 10-22 years after tumour evolution); notably, overall mortality was $4 \%$ due to pituitary carcinomas or neurological complications associated with a rapidly growing tumour (3).

Our patient retained normal PRL for a period of 4 years (during which he had remained on bromocriptine), with the tumour developing aggressive behaviour thereafter and finally presenting with left recurrent laryngeal, hypoglossal and abducens nerve palsies; to our knowledge, such symptomatology has not been reported before. The mechanisms triggering the loss of control and the transformation of tumour phenotype have not been elucidated.

Secondary resistance to DA treatment is a rare phenomenon and presents with an increase in PRL levels and often rapid growth/invasion of the prolactinoma. The real prevalence is unknown and the relevant published literature is limited with only a few cases available $(2,5)$. Before this diagnosis is established, non-compliance to DA needs to be excluded. The pathogenetic mechanisms are not clear and, interestingly, development of late loss of responsiveness to DA has been related with the initiation of gonadal hormone replacement (1). Based on the published literature, the interval for the diagnosis of secondary resistance ranges between 10 months to 15 years (2) and the patients had presented with headache and blurred vision (2), persistent galactorrhoea (6), asymptomatic rise in PRL concentration $(6,7)$ or tumour progression on imaging (8). Notably, secondary resistance has more often been reported in the setting of malignant transformation of a prolactinoma necessitating careful clinical and imaging assessment (9).

In our patient, repeat surgery and/or radiotherapy were not considered as appropriate management strategy and temozolomide was offered. The outcome following the first three cycles was promising with tumour shrinkage and significant reduction in his PRL levels; the optimal response was further confirmed in his latest follow-up 9 months after the initiation of temozolomide. Temozolomide, an alkylating oral chemotherapy agent acting via DNA methylation to induce DNA fragmentation is typically dosed at $150-200 \mathrm{mg} / \mathrm{m}^{2}$ for 5 days in 28 -day cycles; several studies have demonstrated that lack of response after three cycles, either in tumour size or PRL levels, is predictive of a poor outcome $(10,11)$. Review of the literature of case series including five or more patients with pituitary adenomas or carcinomas treated with this agent (9-12 cycles) has shown complete or partial radiological response in $42 \%$ and stable disease in $27 \%$ of them; the response rate in prolactinomas was $44 \%$. Unlike for malignant gliomas, the data on the value of MGMT (Methylguanine-DNA methyltransferase) or of the mismatch repair protein MSH6 tumour status on predicting temozolomide responsiveness are not entirely clear; it should be noted however, that although MGMT expression measured by PCR-based methods has not been associated with response to temozolomide, low MGMT content determined by immunohistochemistry is mostly related with positive response to this agent (11). The optimum duration of temozolomide treatment remains 
uncertain, as does the durability of response following cessation of treatment. Notably, recurrence after cessation of this treatment has been reported in $27-67 \%$ of the patients with various types of pituitary adenomas and variable follow-up duration, with progression-free survival ranging between 8 and 12 months (12).

Interestingly, during his follow-up, our patient was diagnosed with prostatic adenocarcinoma leading to withdrawal of testosterone replacement. Berinder et al. in a population-based matched cohort study in Sweden found reduced prostate cancer risk in males with hyperprolactinaemia (13). The authors suggested that this may be attributed to the history of low androgen exposure as a result of the hyperprolactinaemia-induced hypogonadism. On the other hand, when testosterone replacement is offered to these patients, PSA is monitored more systematically, potentially leading to increased rates of prostate cancer diagnosis and possible bias in the estimation of prostate carcinoma incidence in this group of patients.

Our case represents a rare clinical course of a patient with macroprolactinoma with a remarkably late escape from control and demonstration of invasive/aggressive behaviour, highlights pitfalls in the identification of nonsellar aggressive adenomatous tissue and enhances the value of temozolomide in the management algorithm of such challenging cases.

\section{Declaration of interest}

The authors declare that there is no conflict of interest that could be perceived as prejudicing the impartiality of the research reported.

\section{Funding}

This research did not receive any specific grant from any funding agency in the public, commercial or not-for-profit sector.

\section{Author contribution statement}

Anne de Bray - Specialist Registrar was involved in writing the manuscript. Zaki Hassan-Smith - Consultant Endocrinologist was involved in patient follow-up and in writing the manuscript. Jamal Dirie - medical student was involved in writing the manuscript. Edward Littleton - Consultant Neurologist was involved in patient follow-up. Swarupsinh Chadva - Consultant Neuroradiologist conducted the imaging review and prepared the figures of the manuscript. John Ayuk - Consultant Endocrinologist was involved in patient follow-up. Paul Sanghera - Consultant Oncologist was involved in patient follow-up. Niki Karavitaki - Consultant Endocrinologist was involved in patient follow-up, in writing the manuscript and supervised the preparation of this work. All listed authors contributed to the editing process.

\section{Patient consent}

Written informed consent has been obtained from the patient for publication of the submitted article and accompanying images.

\section{References}

1 Molitch ME. Pharmacologic resistance in prolactinoma patients. Pituitary 20058 43-52. (https://doi.org/10.1007/s11102-005-5085-2)

2 Behan LA, Draman MS, Moran C, King T, Crowley RK, O'Sullivan EP, Smith D, Thompson CJ \& Agha A. Secondary resistance to cabergoline therapy in a macroprolactinoma: a case report and literature review. Pituitary 201114 362-366. (https://doi. org/10.1007/s11102-009-0168-0)

3 Vroonen L, Jaffrain-Rea ML, Petrossians P, Tamagno G, Chanson P, Vilar L, Borson-Chazot F, Naves LA, Brue T, Gatta B, et al. Prolactinomas resistant to standard doses of cabergoline: a multicentre study of 92 patients. European Journal of Endocrinology 2012167 651-662. (https://doi.org/10.1530/EJE-12-0236)

4 Whitelaw BC, Dwarakowska D, Thomas NW, Barazi S, Riordan-Eva P, King AP, Hampton T, Landau DB, Lipscomb D, Buchanan CR, et al. Temozolomide in the management of dopamine agonist-resistant prolactinomas. Clinical Endocrinology 201276 877-886. (https://doi. org/10.1111/j.1365-2265.2012.04373.x)

5 Mallea-Gil MS, Cristina C, Perez-Millan MI, Villafane AM, Ballarino C, Stalldecker G \& Becu-Villalobos D. Invasive giant prolactinoma with loss of therapeutic response to cabergoline: expression of angiogenic markers. Endocrine Pathology 200920 35-40. (https://doi.org/10.1007/s12022-009-9057-3)

6 McCall D, Hunter SJ, Cooke RS, Herron B, Sheridan B \& Atkinson AB. Unusual late development of dopamine agonist resistance in two women with hyperprolatinaemia associated with transition from micro to macroadenoma. Clinical Endocrinology 200766 149-150. (https://doi.org/10.1111/j.1365-2265.2006.02686)

7 Delgrange E, Crabbe J \& Donickier J. Late development of resistance to bromocriptine in a patient with a macroprolactinoma. Hormone Research 199849 250-253. (https://doi.org/10.1159/000023180)

8 Briedahl HD, Topliss DJ \& Pike JW. Failure of bromocriptine to maintain reduction in size of a macroprolactinoma. BMJ 1983287 451-452. (https://doi.org/10.1136/bmj.287.6390.451)

9 Kars M, Roelfsema F, Romijin A \& Pereira AM. Malignant prolactinoma: case report and review of the literature. European Journal of Endocrinology 2006155 523-534. (https://doi.org/10.1530/eje.1.02268)

10 Moisi M, Cruz AS, Benkers T, Rostad S, Broyles FB, Yuen K \& Mayberg M. Treatment of aggressive prolacitn-secreting pituitary adenomas with adjuvant temozolomide chemotherapy: a review. Cureus 201627 e658. (https://doi.org/10.7759/cureus.658)

11 Raverot G, Burman P, McCormack A, Heaney A, Petersenn S, Popovic V, Trouillas J, Dekkers OM \& European Society of Endocrinology. European Society of Endocrinology Clinical Practice Guidelines for the management of aggressive pituitary tumours and carcinomas. European Journal of Endocrinology 2018178 G1-G24. (https://doi.org/10.1530/EJE-17-0796)

12 Halevy C \& Whitelaw BC. How effective is temozolomide for treating pituitary tumours and when should it be used? Pituitary 201720 261-266. (https://doi.org/10.1007/s11102-016-0745-y)

13 Berinder K, Akre O, Granath F \& Hulting AL. Cancer risk in hyperprolactinemia patients: a population-based cohort study. European Journal of Endocrinology 2011165 209-215. (https://doi. org/10.1530/EJE-11-0076) 